\title{
THE SLICE BALANCE APPROACH USING AN ADAPTIVE-WEIGHTED CLOSURE
}

\author{
Michael W. Hackemack \\ Naval Nuclear Laboratory \\ PO Box 1072, Schenectady, NY 12301, USA \\ michael.hackemack@unnpp.gov
}

\begin{abstract}
In this paper, we present a formulation of the slice balance approach using a nonlinear closure relation derived analogously from the adaptive-weighted diamond-difference form of the weighted diamond-difference method for Cartesian grids. The method yields strictly positive solutions that reduce to a standard diamond closure with fine-enough mesh granularity. It can be efficiently solved using Newton-like nonlinear iterative methods with diffusion preconditioning.
\end{abstract}

KEYWORDS: Radiation Transport, Slice Balance Approach, Nonlinear Closure, Positivity Preserving

\section{INTRODUCTION}

It is well known that the discrete ordinates $\left(S_{N}\right)$ transport equation can suffer from negative numerical fluxes due to unrefined spatial discretizations or inadequate Legendre polynomial expansion of the anisotropic scattering source [1,2]. While these negative flux solutions are mathematically valid, they can be extremely undesirable: 1) lead to negative reaction rates in regions of interest (e.g., detector or dose calculation regions); 2) bad oscillatory solution behavior [3] leading to poor weight window generation for use in Monte Carlo calculations; and 3) adversely affect common iterative strategies like nonlinear diffusion acceleration schemes [4]. To counteract the generation of negative fluxes, a variety of methods have been developed including nonlinear moment methods and ad-hoc fixup methods with most only applicable to logically Cartesian grids [2,5].

Strictly Cartesian grids, while being simple to develop and implement efficient transport methods on, can be inefficient and undesirable for a couple of reasons. First, complex and curved geometries need to either be homogenized or approximated with some type of stair-stepping. Second, if certain regions of the problem require finer mesh granularity, then the necessary grid lines must run through the whole domain which can lead to large mesh cell counts (more computational work). Polytopal grids (polygonal or polyhedral mesh cells) as well as unstructured simplicial or tensor cell grids can resolve both of these issues, but require more complex transport discretizations.

The Slice Balance Approach (SBA), based on the multiple-balance approach methodology, is one such method compatible with polytopal grids [6,7]. Mesh cells are decomposed into subregions (slices) with exact spatial moments balance equations and closed with approximate auxiliary equations (i.e., closure relations). However, many of these closures suffer from the generation of negative fluxes based on a mesh cells optical thickness and topology. Inspired by the AdaptiveWeighted Diamond-Difference (AWDD) method for Cartesian cells [5], the presented method utilizes the AWDD closure on an SBA discretization in which each slice acts like a simple slab-like 


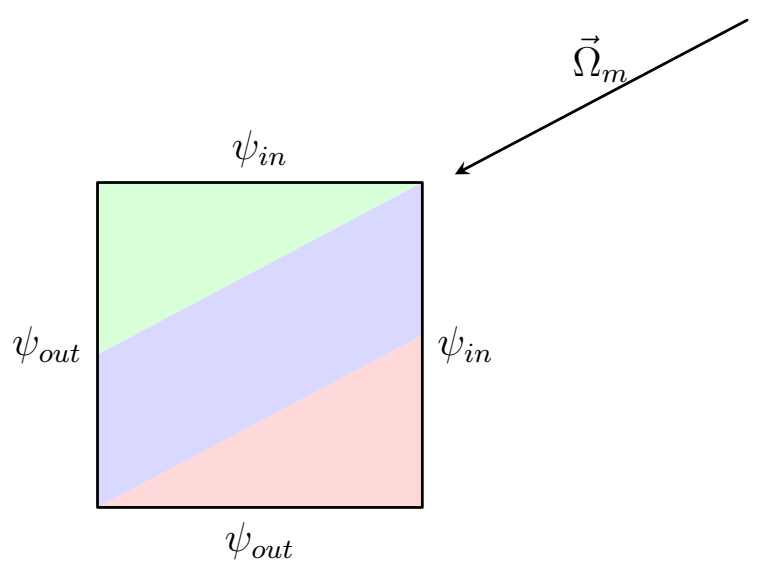

Figure 1: Example slicing of a square into three slices where each slice has a unique incoming face, $\psi_{i n}$, and outgoing face, $\psi_{\text {out }}$.

transport problem. This leads to a method that is efficient on unstructured polytopal mesh cells and eliminates the generation of negative numerical fluxes stemming from unrefined mesh cells.

\section{SLICE BALANCE APPROACH WITH NONLINEAR CLOSURE}

In this section, we present a general framework for our SBA method with slab-like closure relations on each slice. Each mesh cell is decomposed into slices for each ordinate as seen in Figure 1, where each slice has exactly 1 incoming face and 1 outgoing face. Figure 2 gives an example slice on a 2D pentagon (denoted cell $K$ ) for an ordinate $\vec{\Omega}_{m}$. On the slice, an exact balance equation analogous to the weighted diamond difference (WDD) can be formulated and is given by

$$
\mathcal{P}_{K, s}\left(\psi_{m, K, s}^{+}-\psi_{m, K, s}^{-}\right)+\sigma_{t, K} \bar{\psi}_{m, K, s}=q_{m, K},
$$

where $\mathcal{P}_{K, s}=A_{s} / V_{s}$ is the project slice area. $A_{s}$ is the slice area denoted in Figure $2 \mathrm{~b}$ as its width, and $V_{s}$ is the slice volume denoted in Figure 2a by the shaded region. Using the WDD methodology, a closure relation for Eq. (1) can be written as

$$
\bar{\psi}_{m, K, s}=\frac{1}{1+a_{s}} \psi_{m, K, s}^{+}+\frac{a_{s}}{1+a_{s}} \psi_{m, K, s}^{-}
$$

where $a_{s}$ is the weighting factor for slice $s$. If $a_{s}$ remains constant, then the method would be linear ( $a=0$ and $a=1$ correspond to the standard step and diamond-difference closures, respectively). Indeed, we denote SBA-DD and SBA-S as the SBA method utilizing the diamond-difference and step closures, respectively. Instead, using the AWDD closure (which we denote as SBA-AWDD) encompasses a nonlinear predictor-corrector scheme. Using Eq. (2), the predictor step uses the diamond closure to solve for the average flux in slice $s, \bar{\psi}_{m, K, s}^{p}$, with 


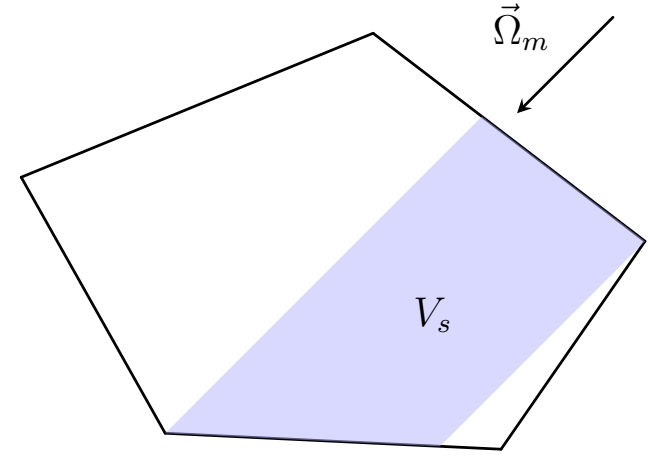

(a) Global cell and slice

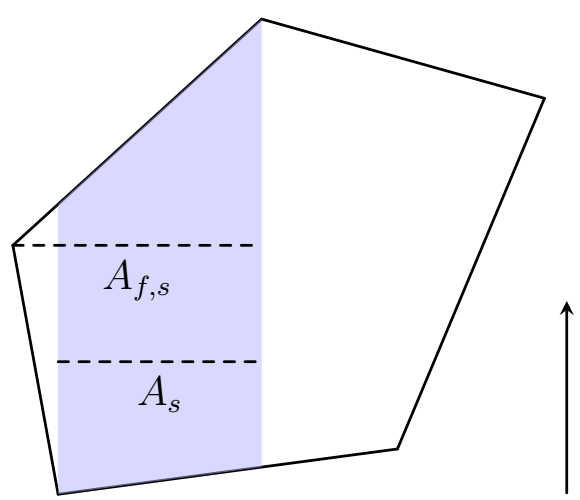

$(0,1)$

Figure 2: Demonstrative polygonal cell and singular slice (left) which is then rotated so that the angle aligns with $(0,1)$ (right).

$$
\bar{\psi}_{m, K, s}^{p}=\frac{q_{m, K}+2 \mathcal{P}_{K, s} \psi_{m, K, s}^{-}}{\sigma_{t, K}+2 \mathcal{P}_{K, s}}
$$

Then, a corrected weighting factor can be computed with

$$
a_{s}^{c}=\min \left(1,\left|\frac{\bar{\psi}_{m, K, s}^{p}}{2\left(\psi_{m, K, s}^{-}-\bar{\psi}_{m, K, s}^{p}\right)}\right|\right),
$$

which provides a sufficient condition to ensure outgoing positivity. Once $a_{s}^{c}$ is computed, the corrected average flux $\bar{\psi}_{m, K, s}^{c}$ along with the outgoing flux of slice $s$ are given by

$$
\bar{\psi}_{m, K, s}^{c}=\frac{q_{m, K}+\left(1+a_{s}^{c}\right) \mathcal{P}_{K, s} \psi_{m, K, s}^{-}}{\sigma_{t, K}+\left(1+a_{s}^{c}\right) \mathcal{P}_{K, s}} \quad \text { and } \quad \psi_{m, K, s}^{+}=\left(1+a_{s}^{c}\right) \bar{\psi}_{m, K, s}^{c}-a_{s}^{c} \psi_{m, K, s}^{-}
$$

respectively. Then, the average cell $K$ flux of ordinate $m$ and the outgoing flux of face $f$ are

$$
\bar{\psi}_{m, K}=\sum_{s \in K} \frac{V_{s}}{V_{K}} \bar{\psi}_{m, K, s}^{c} \quad \text { and } \quad \psi_{m, K, f}^{+}=\sum_{s \in f^{+}} \mathcal{A}_{f, s}^{+} \psi_{m, K, s}^{+}
$$

respectively, where $s \in f^{+}$denotes all slices for which face $f$ is outgoing, $\mathcal{A}_{f, s}^{+}=A_{s} / A_{f, s}$ is the projected area of slice $s$ for the outgoing face $f$, and $V_{K}$ is the volume of cell $K$.

We note that the SBA geometric quantities were detailed using the 2D example given in Figure 2. Analogues are straightforward for 1D and 3D. In 1D, SBA reduces to exactly the standard WDD method. In 3D, the cell can be analogously rotated so that the angle aligns with $(0,0,1)$. Then, the slice is determined by the intersection of polygons comprising the incoming/outgoing faces projected onto the $x y$ plane. 


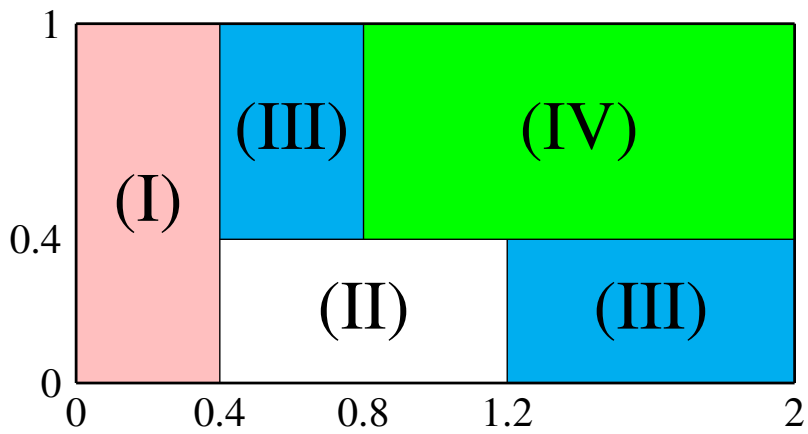

\begin{tabular}{|c|c|c|c|}
\hline Region & $\sigma_{t}$ & $\sigma_{s}$ & $Q$ \\
\hline (I) & 1.0 & 0.0 & 1.00 \\
(II) & $10^{-4}$ & 0.0 & 0.00 \\
(III) & 100.0 & 0.0 & 0.00 \\
$(\mathrm{IV})$ & 10.0 & 9.999 & 0.00 \\
\hline
\end{tabular}

Figure 3: Configuration of the 2D shielding problem where $(\mathrm{X})$ denotes the region number.

\section{SOLUTION STRATEGIES}

In this section, we briefly outline some solution strategies that can be employed to solve the nonlinear SBA-AWDD scheme. In a previous work, we analyzed iterative strategies for converging nonlinear $S_{N}$ discretizations [8]. Effective strategies were demonstrated using combinations of Newton-like nonlinear iterative solvers and physics based preconditioning. Therefore, we analyze SBA-AWDD using the Richardson, Broyden, nonlinear Krylov acceleration (NKA), and Jacobianfree Newton-Krylov (JFNK) methods which has a common iterative form: $x^{(\ell+1)}=x^{(\ell)}+\Delta x^{(\ell)}$, where the differences lie in the determination of the update vector, $\Delta x^{(\ell)}$. The Broyden, NKA, and JFNK methods utilize subspaces of previous solution states/residuals in their iterative procedures (JFNK's subspaces are used in its inner GMRES solver). For a subspace of size $n$, Broyden and JFNK utilize $n+O(1)$ global vectors and handle their memory storage by restarting. On the other hand, NKA uses $2 n+O(1)$ global vectors and a reduced memory approach where the oldest vectors are removed when the vector space reaches its maximum allowed size.

Along with these nonlinear iterative solvers, physics-based preconditiong can be applied (i.e., diffusion acceleration). Since the SBA schemes analyzed in this work yield only average solutions on a mesh cell, we utilize cell-based diffusion preconditioning [9]: linear diffusion synthetic acceleration (DSA), nonlinear diffusion acceleration (NDA), and its partial-current variant (pNDA). The interested reader should review [8] for more complete implementation details which we will not provide here for brevity.

\section{NUMERICAL RESULTS}

We now present some numerical results for our SBA-AWDD method using a monoenergetic, heterogeneous 2D shielding-type problem with source, absorbing, near-void, and scattering regions. The geometry and material properties are given in Figure 3. Each boundary is a vacuum condition, and a $S_{8}$ level-symmetric quadrature is used. Negative fluxes arise in both of the absorbing regions (i.e., Region (III)). Polygonal meshes are formed with the PolyMesher software [10], and then cut lines are added through the problem to preserve material boundaries. Example solutions are presented on a log scale in Figure 4 for SBA-DD and SBA-AWDD where white space indicates a mesh cell with negative solution, thus demonstrating the SBA-AWDD method's ability to preserve 


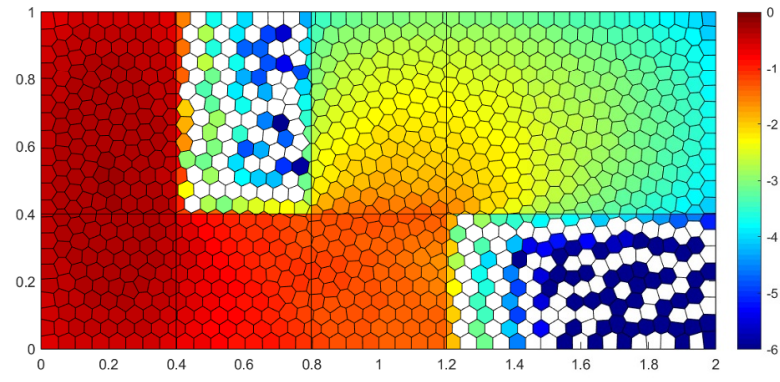

(a) SBA-DD

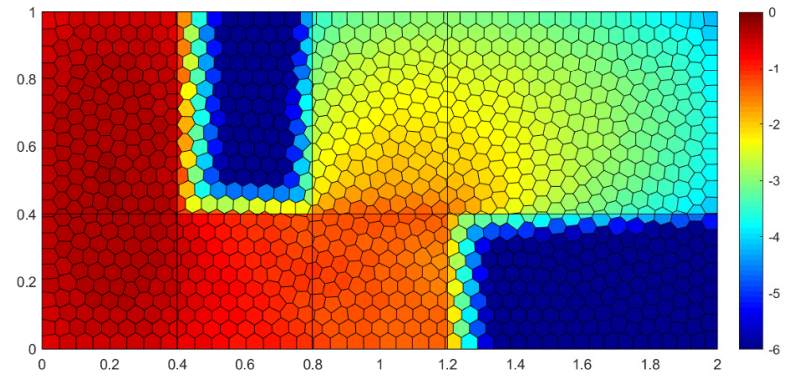

(b) SBA-AWDD

Figure 4: Example solutions plotted on a log scale of the SBA method using the (a) diamond closure and the (b) AWDD closure. White space indicates a cell with a negative solution.

strictly-positive flux solutions. Also, the flux along the diagonal from $(0,1)$ to $(2,0)$ for the diamond, AWDD, and step closures is plotted on a log scale in Figure 5 against a reference solution generated on a $320 \times 160$ Cartesian grid using SBA-DD. The gaps in the flux using the diamond closure correspond to mesh cells with negative solutions. We can see that SBA-AWDD provides much more accurate solutions in the absorbing regions. Likewise, SBA-DD yields unphysical and inaccurate oscillations, and SBA-S provides a strictly positive solution but with significantly more numerical diffusion.

Next, we provide some analysis on the error by monitoring the convergence of the average flux in the scattering region (i.e., Region (IV)). We utilize both uniform $h$-refinement on Cartesian cells and adaptive mesh refinement (AMR) utilizing the methodology of Hackemack and Ragusa [11]. The initial mesh $(10 \times 5)$ contains only quadrilateral cells, but refined meshes do not make use of hanging nodes and instead form degenerate polygons. A heuristic a posteriori local error indicator for cell $K$ monitors the interfacial jumps:

$$
\eta_{K}=\int_{\partial K} \llbracket \phi \rrbracket^{2}=\int_{\partial K}\left(\sum_{m} w_{m} \llbracket \psi_{m} \rrbracket\right)^{2}
$$

where $\llbracket \cdot \rrbracket$ is the jump operator along a face: $\llbracket \phi(\vec{r}) \rrbracket=\phi^{+}(\vec{r})-\phi^{-}(\vec{r})$, with $\phi^{ \pm}(\vec{r})=\lim _{s \rightarrow 0^{ \pm}} \phi(\vec{r}+s \vec{n})$. With the error estimates defined, the criterion to determine the cells flagged for refinement is

$$
\eta_{K} \leq \alpha \max _{K^{\prime}}\left(\eta_{K^{\prime}}\right)
$$

where $\alpha$ is a user-defined value $(0,1)$ which we set to 0.5 in this work. Figure 6 provides the relative error in Region (IV) using uniform and adaptive AMR for SBA using the diamond, AWDD, and step closures. The reference solution was generated using SBA-DD on a $5120 \times 2560$ Cartesian grid. Under uniform refinement, all three methods converge at a rate of $h^{2}$ since SBA averages the angular flux across slices (see [6] for more details on SBA convergence rates), but SBA-DD and 


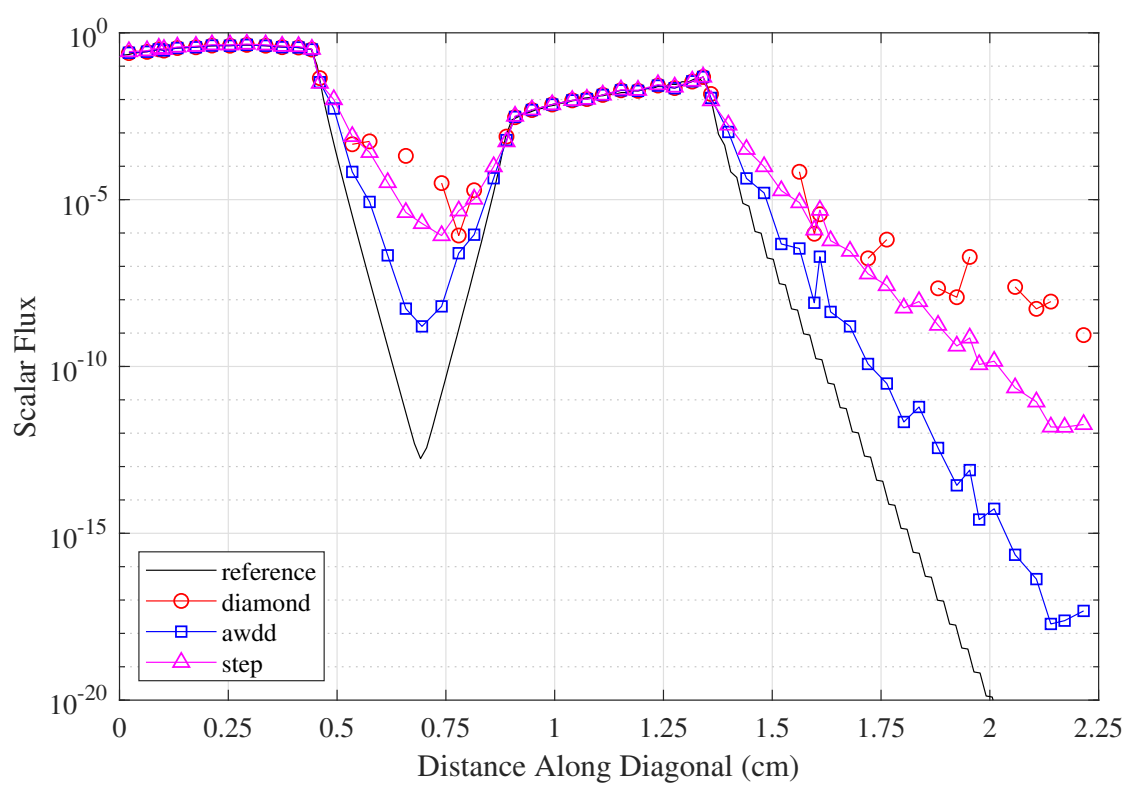

Figure 5: Scalar flux solution for different SBA closures along the domain diagonal of a 1,024 cell (pre-split) polygonal mesh. The reference solution was generated with SBA-DD on a 320x160 Cartesian Grid.

SBA-AWDD provide greater error reduction over SBA-S. We can also see that SBA-DD and SBAAWDD converge to the same solution under fine enough mesh granularity as we would expect. Spatial adaptivity provides improved error convergence over uniform refinement using the same number of degrees of freedom.

Finally, we seek to provide some results demonstrating the required iterative work to converge the AWDD solution seen in Figure 4b. Table 1 gives the number of transport sweeps required to terminate the simulation at $\|\mathbf{F}(x)\|_{2} \leq 10^{-8}\left\|\mathbf{F}\left(x^{(0)}\right)\right\|_{2}$, where the initial solution, $x^{(0)}$, is simply an all-zero vector. The Broyden, NKA, and JFNK methods are analyzed with subspace sizes of 5, 10, 20, and 30. The Richardson method is analyzed for comparative purposes. Preconditioning using DSA, NDA, and pNDA is also used, and the cases listed as 'None' correspond to no physics-based preconditioning being applied. We can see good reduction in sweep numbers when using Broyden/NKA/JFNK with no diffusion preconditioning and Richardson with diffusion preconditioning. Even further reduction is realized with Broyden/NKA/JFNK using diffusion preconditioning. These results are similar to those previously reported using different nonlinear $S_{N}$ discretizations [8].

\section{CONCLUSIONS}

In this work, we have presented a $S_{N}$ transport discretization using the slice balance approach with a nonlinear, adaptive-weighted closure relation. For a given ordinate, a mesh cell is decomposed into subregions (slices) where a balance equation analogous to the adaptive-weighted diamonddifference closure for the weighted diamond-difference method is formed. A monoenergetic, het- 


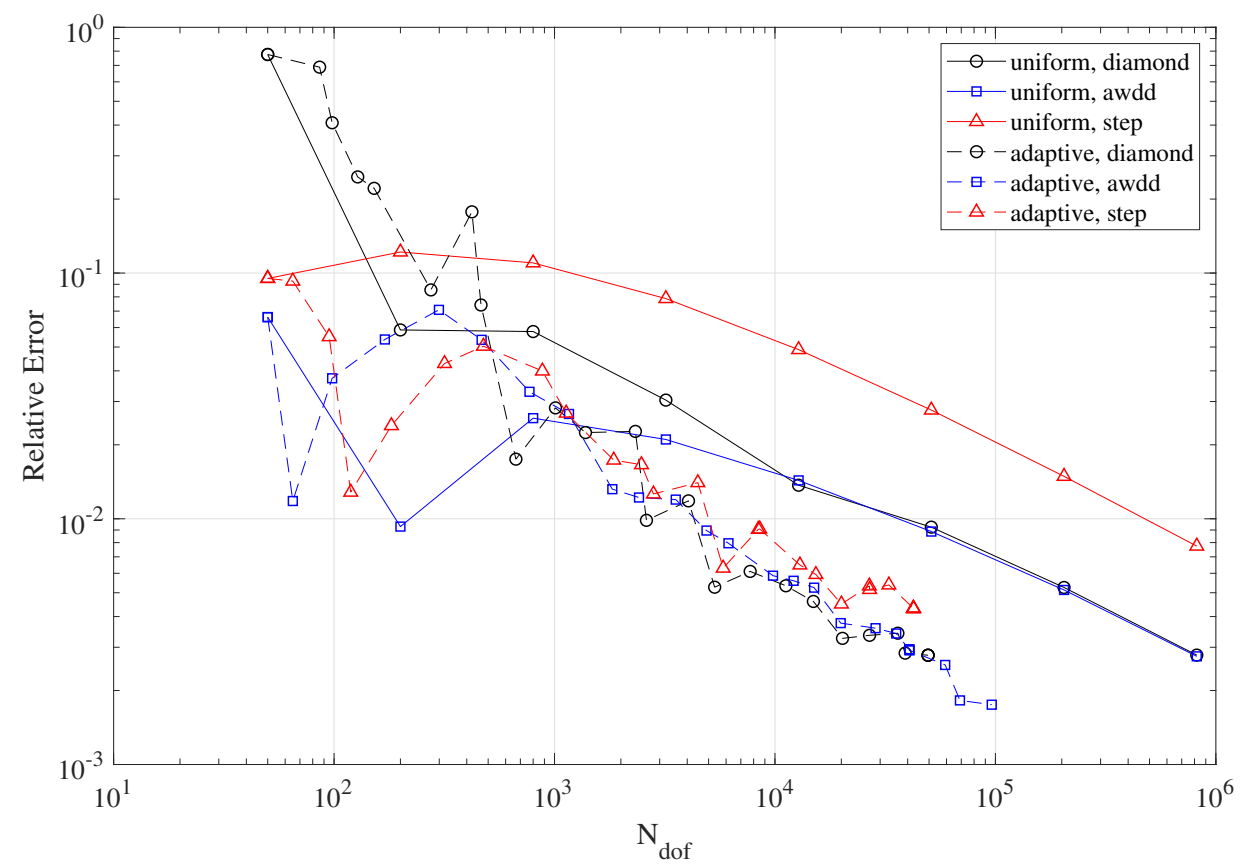

Figure 6: Convergence of the average flux in Region (IV) using uniform and adaptive $h$-refinement.

Table 1: Number of sweeps needed for SBA-AWDD for the 2D Shielding Problem.

\begin{tabular}{cccccc} 
Method & Subspace & None & DSA & NDA & pNDA \\
\hline Richardson & - & 154 & 19 & 16 & 18 \\
\hline \multirow{4}{*}{ Broyden } & 5 & 34 & 12 & 10 & 11 \\
& 10 & 33 & 11 & 10 & 10 \\
& 20 & 29 & 11 & 10 & 10 \\
\hline \multirow{3}{*}{ NKA } & 30 & 30 & 11 & 10 & 10 \\
\hline \multirow{5}{*}{ JFNK } & 5 & 33 & 11 & 10 & 10 \\
& 10 & 25 & 10 & 10 & 9 \\
& 20 & 23 & 10 & 10 & 9 \\
& 30 & 29 & 10 & 10 & 9 \\
\hline & 5 & 29 & 13 & 12 & 12 \\
& 20 & 29 & 13 & 12 & 12 \\
\hline
\end{tabular}


erogeneous numerical problem is analyzed. It is shown that SBA-AWDD yields strictly positive flux solutions and provides reduced error compared to SBA with diamond and step closures. The performance of different iterative strategies was also analyzed, and it was shown that Newton-like nonlinear iterative solvers with diffusion preconditioning greatly reduce the number of required transport sweeps.

\section{ACKNOWLEDGMENTS}

The submitted manuscript has been authored by a contractor of the U.S. Government under contract No. DOE-89233018CNR000004. Accordingly, the U.S. Government retains a non-exclusive, royalty-free license to publish or reproduce the published form of this contribution, or allow others to do so, for U.S. Government purposes.

\section{REFERENCES}

[1] E. Lewis and W. Miller. Computational Methods of Neutron Transport. John Wiley and Sons, Inc., New York, NY (1984).

[2] K. Lathrop. "Spatial differencing of the transport equation: positivity vs. accuracy." Journal of Computational Physics, volume 4, pp. 475-498 (1969).

[3] P. B.G. and A. Haghighat. "Analysis of inherent oscillations in multidimensional $S_{N}$ solutions of the neutron transport equation." Nuclear Science and Engineering, volume 124(1), pp. 31-62 (1996).

[4] K. Smith and J. Rhodes III. "Full-core 2-D LWR core calculations with CASMO-4E." In Proc. Int. Conf. New Frontiers of Nuclear Technology: Reactor Physics, Safety, and HighPerformance Computing, pp. 7-10 (2002).

[5] R. Alcouffe. "An Adaptive Weighted Diamond Differencing Method for Three-Dimensional XYZ Geometry." Transactions of the American Nuclear Society, volume 68, pp. 206-212 (1993).

[6] R. Grove. A Characteristic-Based Multiple Balance Approach for Solving the $S_{N}$ Equations on Arbitrary Polygonal Meshes. Ph.D. thesis, University of Michigan (1996).

[7] R. Grove. "The Slice Balance Approach (SBA): A Characteristic-Based, Multiple Balance $S_{N}$ Approach on Unstructured Polyhedral Meshes." In M\&C 2005: International Topical Meeting on Mathematics and Computation, Supercomputing, Reactor Physics, and Nuclear and Biological Applications (2005).

[8] M. Hackemack. "Solving Nonlinear Discretizations of $S_{N}$ Transport Calculations." Annals of Nuclear Energy, volume 137 (2020).

[9] K.-S. Kim and M. DeHart. "Unstructured partial- and net-current based coarse mesh finite difference acceleration applied to the extended step characteristics method in NEWT." $A n$ nals of Nuclear Energy, volume 38, pp. 527-534 (2011).

[10] C. Talischi, G. H. Paulino, A. Pereira, and I. Menezes. "PolyMesher: a general-purpose mesh generator for polygonal elements written in Matlab." Structural and Multidisciplinary Optimization, volume 45(3), pp. 309-328 (2012).

[11] M. Hackemack and J. Ragusa. "Quadratic serendipity discontinuous finite element discretization for $S_{N}$ transport on arbitrary polygonal grids." Journal of Computational Physics, volume 374, pp. 188-212 (2018). 